\title{
Cautionary Guidelines for Machine Learning Studies with Combinatorial Datasets
}

Andrew F. Zahrt, Jeremy J. Henle, and Scott E. Denmark* sdenmark@illinois.edu

Roger Adams Laboratory, Department of Chemistry, University of Illinois Urbana, Illinois, 61801

\section{SUPPORTING INFORMATION}

Table of Contents

Information Regarding Dataset

Modeling Information

Statistical Analysis

References 


\section{Information Regarding Dataset}

The dataset used in this study is the same as gathered in the original report, which contains the full dataset, descriptors, and link to the gitlab repository. ${ }^{1}$ Further, a full description of the original descriptor sets (ASO, SIF, and MIF descriptors) is identical to those published previously. ${ }^{2}$ Descriptors are calculated by first generating a conformer distribution for every member of the in silico library of catalyst candidates. The in silico library of BINOL-phosphoric acids used in this study contains 806 members. Every conformer for every catalyst is then superimposed with respect to a common core scaffold and placed in a common grid. The ASO descriptors are then generated from the collection of conformers for a given molecule. For each conformer, every grid point is queried if it falls within the van der Waals radius of an atom in the molecule and assigned a binary value: yes $=1$, no $=0$. This process is repeated for every conformer for a given molecule. Thus, if an individual catalyst candidate has $n$ conformers, possible values at each grid point range from 0 to $n$ for that molecule. The occupancy values at each grid point are then normalized to the number of conformers, so that every grid point contains a value between 0 and 1 . These numbers are the ASO descriptors. In this case, the ASO describes only the steric properties of the molecules. This parameterization was compared with two different single-conformer representations. The first is a simple steric indicator field (SIF). In this case, the calculation protocol is identical to the ASO, except only one conformer of a catalyst is used in the calculation rather than an ensemble of conformers. Thus, all descriptors are either 1 or 0 in the indicator field. The second is the use of electronic and steric MIFs, as employed in Comparative Molecular Field Analysis (CoMFA). ${ }^{3}$ In this case, a steric MIF was calculated using Lennard-Jones potentials with an sp $^{3}$-hybridized carbon atom as a probe atom at each grid point, and an electronic MIF was calculated using the electrostatic potential energy at each grid point using a positron as a probe. For each single- 
conformer representation, a $C_{2}$-symmetric conformer of the molecule was selected for minimization such that each catalyst structure used occupied similar relative conformations. These structures were then minimized at the PM6 level of theory. In this way, differences in descriptor profiles will be attributed to structural differences rather than a difference in the relative conformation of the structures.

The identity of training and test compounds is given in the following figures (Figure S1S4). Note that this partitioning is the designation of out of sample predictions; for the random partitioning, all possible 1075 reactions were used for random selection of the external test set. The model reaction used in this study is depicted in Figure S1:

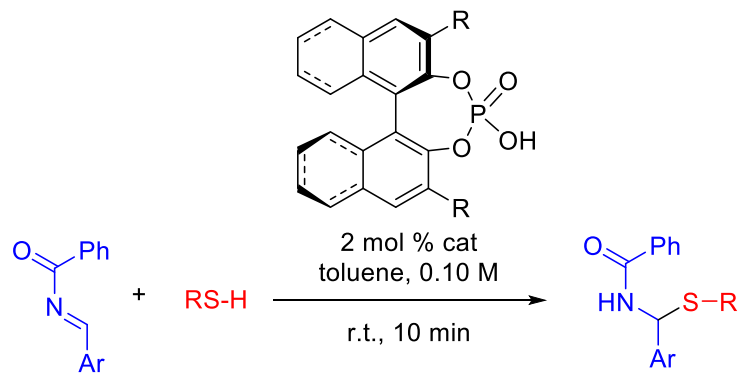

Figure S1. Enantioselective addition of thiols to imines.

The different possible substrate combinations are depicted in Figure S2. Substrate combinations used for training and cross validation are those incorporating imines 1-4 and thiols A-D. These are the substrate combinations used in the top left quadrant or bottom left quadrant of Figure 2 in the main text depending on which catalyst structure they are paired with. Substrate combinations incorporating imine 5 or thiol $\mathrm{E}$ are used in the external test set, are compose reactions in either the top right or bottom right quadrant of Figure 2 depending on which catalyst they are paired with. 


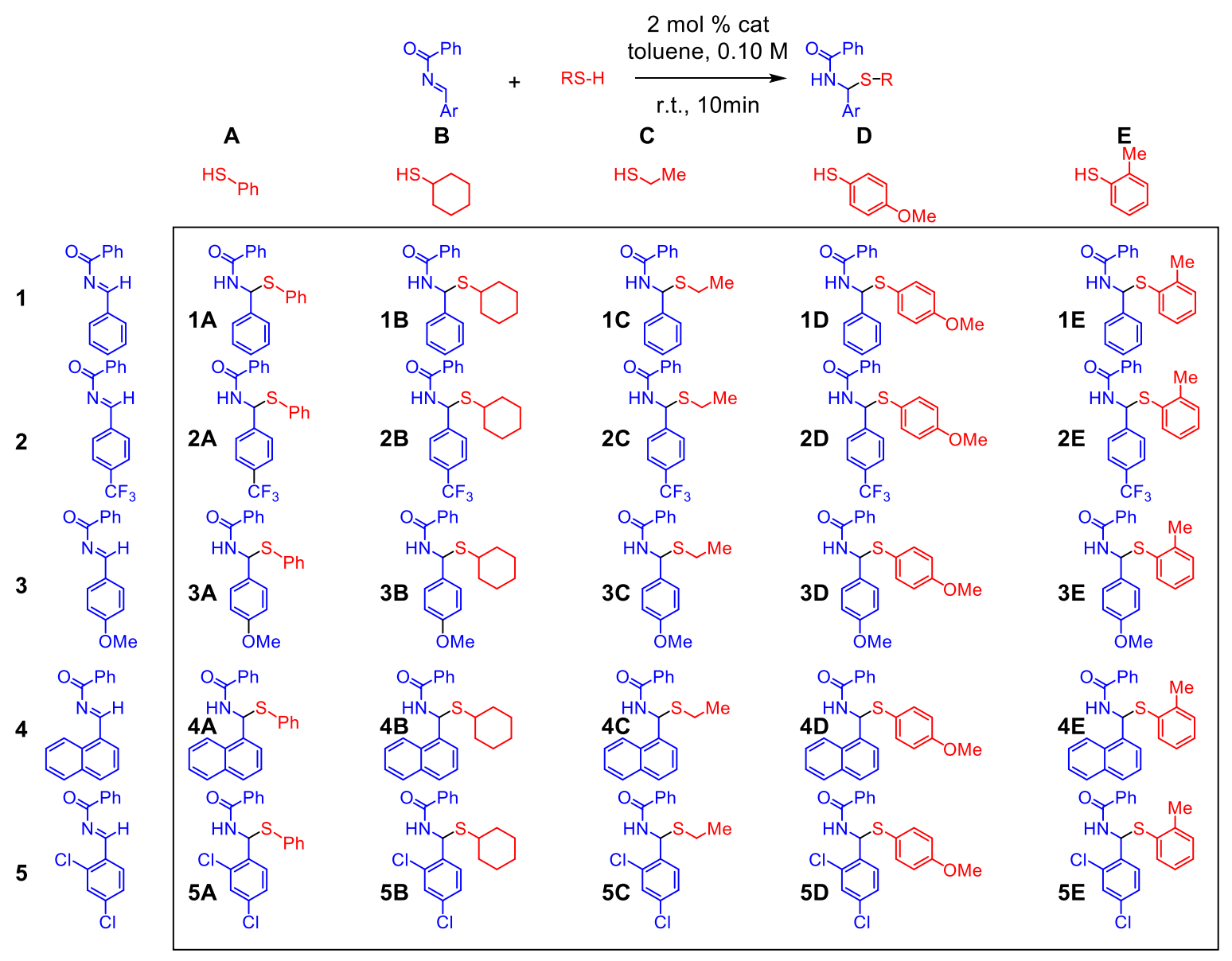

Figure S2. The 25 unique substrate combinations used in this study. Products incorporating imines 1-4 and thiols A-D are designated as training substrate combinations, whereas products incorporating imine 5 or thiol $\mathrm{E}$ are used as out of sample predictions in the external test set.

The 24 training set catalysts are depicted in Figure S3. These are the catalyst structures used in either the top left or top right quadrants of Figure 2 in the main text depending on which substrate combination they are paired with. 


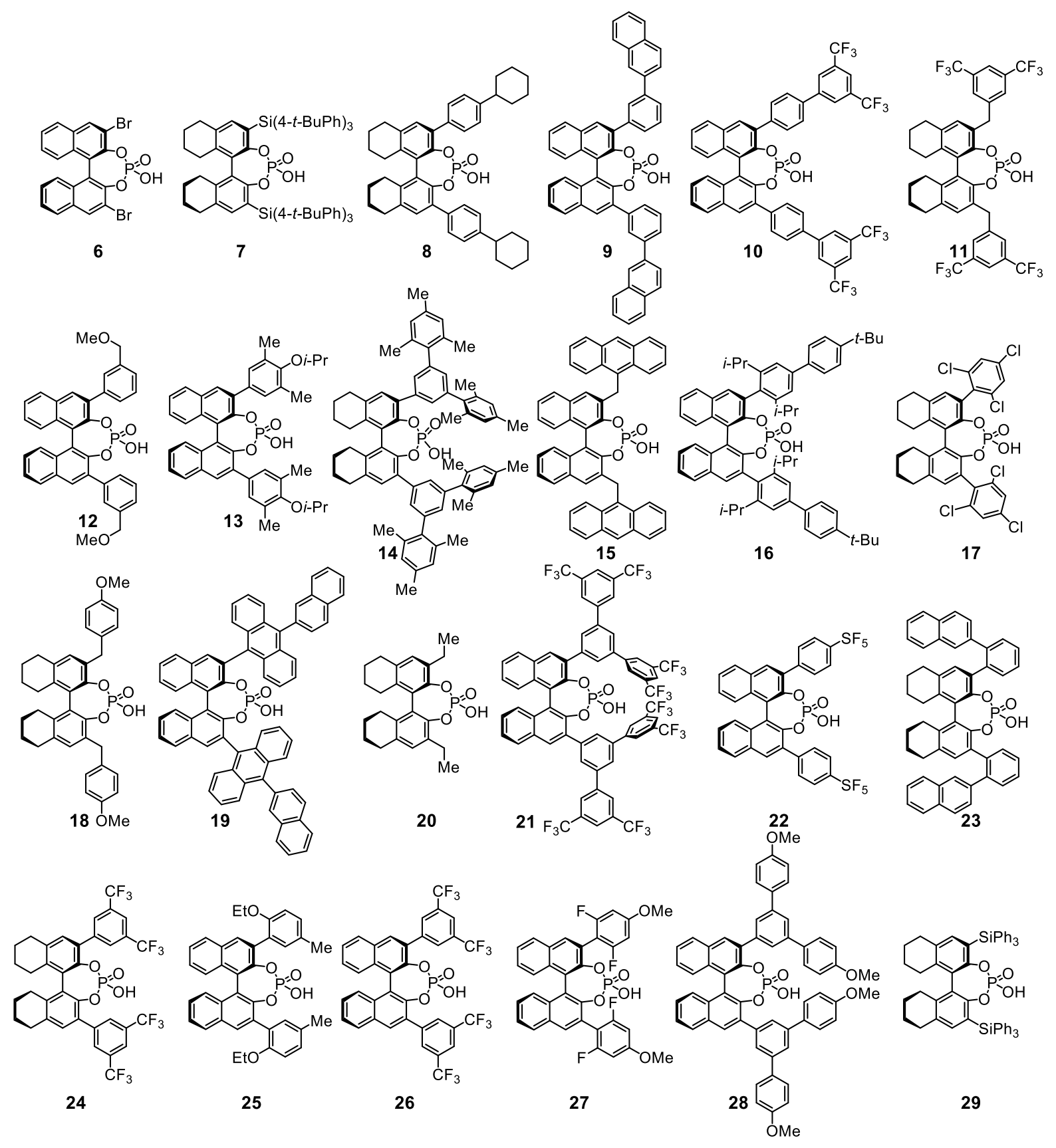

Figure S3. Catalysts selected by the Kennard-Stone algorithm used for model training and cross validation in the non-random data partitioning.

The out of sample catalyst structres used in the external test set are given if Figure S4. These catalysts are used to construct reactions in either the bottom left or bottom right quadrants of Figure 2 in the main text depending on which substrate combination they are paired with. 


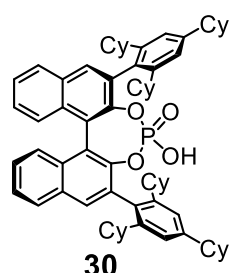

30

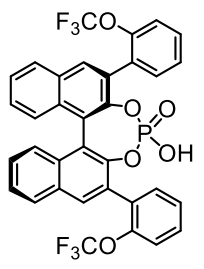

35

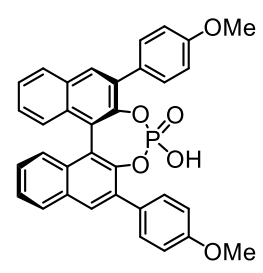

40<smiles>O=P(O)(O)OCc1c(-c2c(OP(=O)(O)O)c(Cc3ccc(C(F)(F)F)cc3C(F)(F)F)cc3ccccc23)c(Cc2ccc(C(F)(F)F)cc2C(F)(F)F)cc2ccccc12</smiles>

45
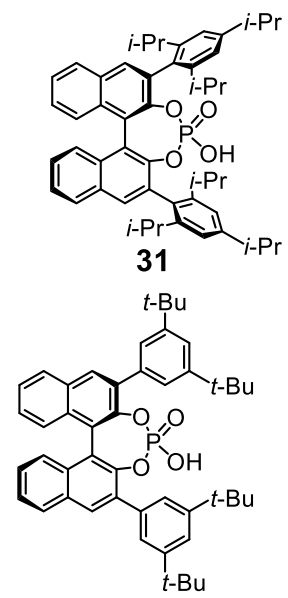

36
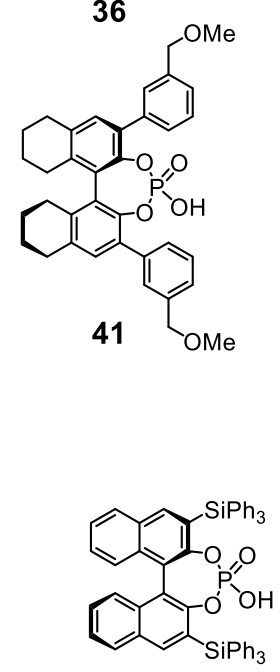

46

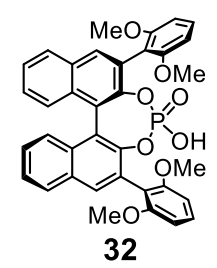

$t-\mathrm{Bu}$

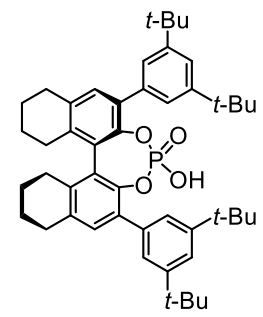

37

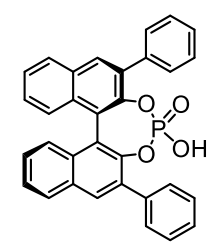

42

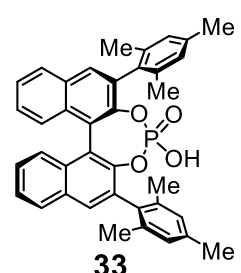

33

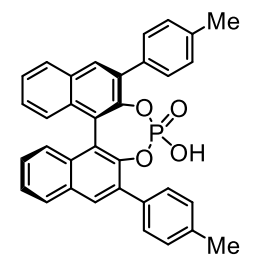

38

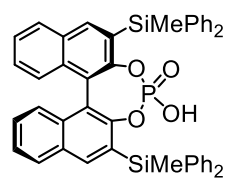

43

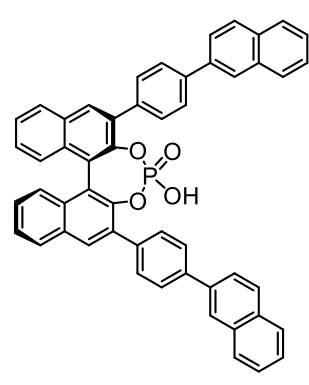

47
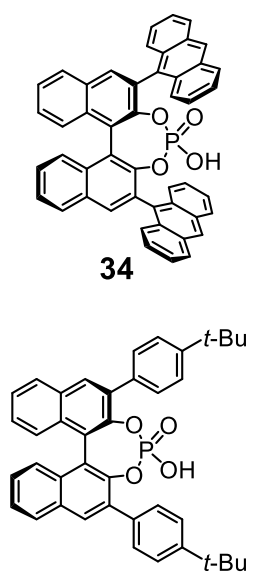

39

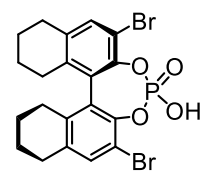

44

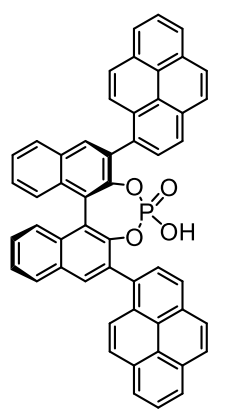

48

Figure S4. Catalysts used to construct out of sample predictions in the external test set.

As previously stated, all 1075 reactions were used in the random partitioning method. Further, the random partitioning was repeated 10 times to ensure these was no difference in model performance depending on which reactions were selected as training data. There was no significant different between models in randomply partitioned data, so only one was presented in the text for ease of presentation. The hyperparameterization and cross validation results are available for all 10 repeats in the associated csv files. 


\section{Modeling Information.}

Prior to modeling, descriptors were scaled and subjected to a varance threshold of 1 . Then, f-regression was used to select the best 109 best descriptors. The number 109 was selected because that was the number of features left in the ASO after applying the varience threshold. Thus, no descriptors were removed from the ASO with f-regression, whereas for the SIF and MIF descriptors some were removed so the models had consistent dimensionality. It is worth noting that for the SIF and MIF descriptors with gradient boosting models that this gave optimal results in an independent study. ${ }^{2}$ All preprocessing and subsequent modeling was performed using SciKit Learn. ${ }^{4}$

In the case of the molecular field descriptors (ASO, SIF, MIF), the dimensionality of the input is reduced to 109 descriptors first by applying a variance threshold and then by selecting the best descriptors as determined using f-regression. For the random featurization, each catalyst is represented by a vector of 91 random numbers, each imine is represented by a vector of 9 random numbers, and each thiol is represented by a vector of 9 random numbers (in all cases, the number range from -1 to 1$).{ }^{5}$ These vectors are concatenated to construct the total reaction parameters, thus every reaction with the same catalysts will have the same set of 91 random numbers. Finally, 1hot encoding indicates the presence of absence of a chemical entity with bit vectors (binary data, 0 and 1). Because there are 43 catalysts, a catalyst is represented by 43 variables, which will be all 0 except for one variable corresponding to the presence of that catalyst, designated by a 1 . The same is done for imines, which uses bit vectors with five variables, and thiols, which also uses bit vectors with five variables. Each bit vector is concatenated, so each reaction is represented with a bit vector containing 53 variables. Each vector will have 50 zeros and threes ones, and the indices of the non-zero values in the vector represents which chemical entities are present. 
Gradient boosting regression was selected as the modeling method used in this approach because it had the most similar performance across molecular field type descriptors in our previous study. ${ }^{2}$ Gradient boosting is also a tree-based approach that builds the ensemble sequentially, adding estimators to compensate for shortcomings in the existing model. A random optimization of hyperparameters was used. In this work, hyperparameters optimized include min_samples_split, ranging from 0.1 to 0.5 , max_features, ranging from 0 to 1 , max_depth, ranging from 1 to 50 , subsample from 0 to 1 , learning_rate, ranging from 0 to 1 , and n_estimators, ranging from 1 to 1000. Hyperparameter selection was evaluated by 10 -fold cross validation, and 500 iterations were used in optimization. Full results from the hyperparameter search can be found in the accociated excel files.

\section{Statistical Analysis.}

A full summary of the statistical analysis is given in this section. To compare the mean errors of the different modeling types, 1-way ANOVAs were performed for each data partitioning methods. Each ANOVA has 5 levels: ASO, SIF, MIF, random featurization, and 1-hot. All statistic were performed with OriginPro software.

Analysis for randomly partitioned data.

Descriptive Statistics
\begin{tabular}{|r|r|r|c|r|r|}
\hline & N Analysis & N Missing & Mean & Standard Deviation & SE of Mean \\
\hline aso & 691 & 0 & 0.16885 & 0.17698 & 0.00673 \\
\hline sif & 691 & 0 & 0.16325 & 0.16878 & 0.00642 \\
\hline mif & 691 & 0 & 0.17395 & 0.17694 & 0.00673 \\
\hline random & 691 & 0 & 0.18561 & 0.18767 & 0.00714 \\
\hline 1-hot & 691 & 0 & 0.28276 & 0.28298 & 0.01077 \\
\hline
\end{tabular}

Figure S5. Descriptive statistics for the MADs of each group for the randomly selected test sets. 


\begin{tabular}{|c|c|c|c|c|c|c|c|c|}
\hline & MeanDiff & SEM & q Value & Prob & Alpha & Sig & $\overline{\mathrm{LCL}}$ & UCL \\
\hline sif aso & 0.05856 & 0.02029 & 4.08096 & 0.03193 & 0.05 & 1 & 0.0032 & 0.11392 \\
\hline mif aso & 0.08757 & 0.02029 & 6.10257 & $1.55189 \mathrm{E}-4$ & 0.05 & 1 & 0.03221 & 0.14293 \\
\hline mif sif & 0.02901 & 0.02029 & 2.02161 & 0.60852 & 0.05 & 0 & -0.02635 & 0.08437 \\
\hline random aso & 0.21985 & 0.02029 & 15.32075 & $2.22045 \mathrm{E}-16$ & 0.05 & 1 & 0.1645 & 0.27521 \\
\hline random sif & 0.16129 & 0.02029 & 11.23979 & 1.93179E-14 & 0.05 & 1 & 0.10593 & 0.21665 \\
\hline random mif & 0.13228 & 0.02029 & 9.21818 & $7.11126 \mathrm{E}-10$ & 0.05 & 1 & 0.07692 & 0.18764 \\
\hline 1-hot aso & 0.12991 & 0.02029 & 9.05291 & 1.53909E-9 & 0.05 & 1 & 0.07455 & 0.18527 \\
\hline 1-hot sif & 0.07135 & 0.02029 & 4.97195 & 0.00401 & 0.05 & 1 & 0.01599 & 0.12671 \\
\hline 1-hot mif & 0.04234 & 0.02029 & 2.95034 & 0.22596 & 0.05 & 0 & -0.01302 & 0.0977 \\
\hline 1-hot random & -0.08994 & 0.02029 & 6.26784 & $9.12664 \mathrm{E}-5$ & 0.05 & 1 & -0.1453 & -0.03459 \\
\hline
\end{tabular}

Sig equals 1 indicates that the difference of the means is significant at the 0.05 level.

Sig equals 0 indicates that the difference of the means is not significant at the 0.05 level.

Figure S6. Tukey post hoc test with pairwise comparisons between groups for the randomly selected test sets.

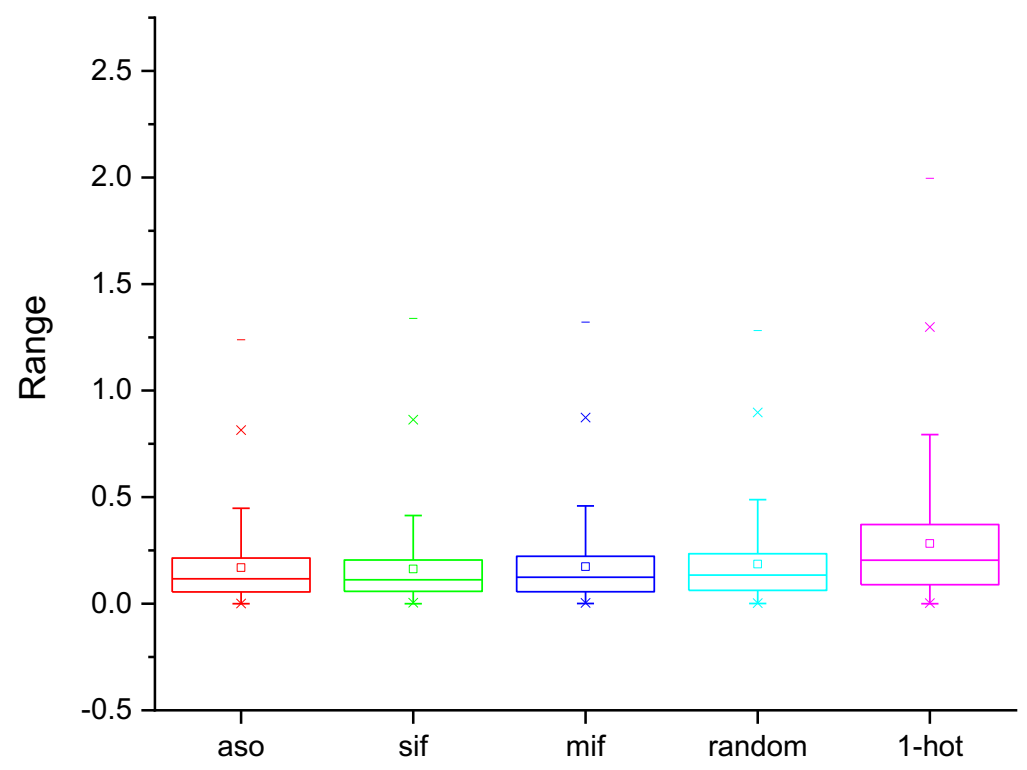

Figure S7. Box plots of MADs for each group for the randomly selected test sets. 


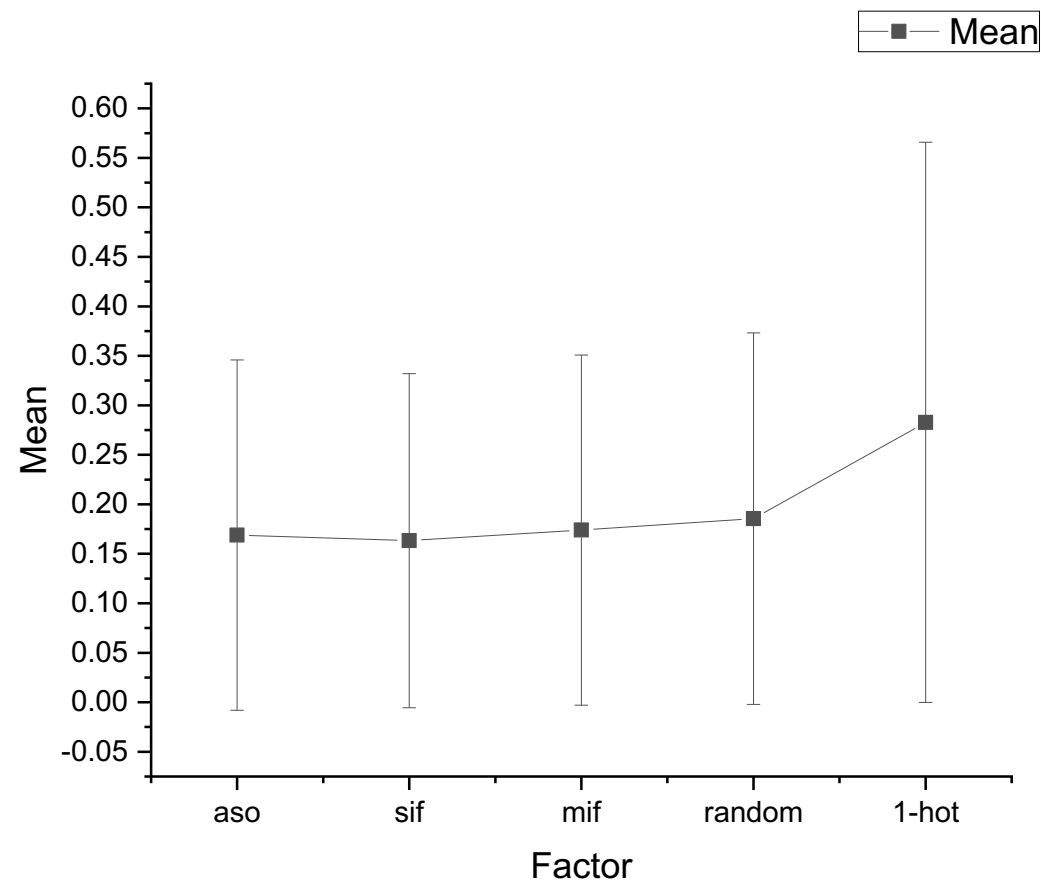

Figure S8. Means plot for the 5 levels used in the ANOVA for the randomly selected test sets.

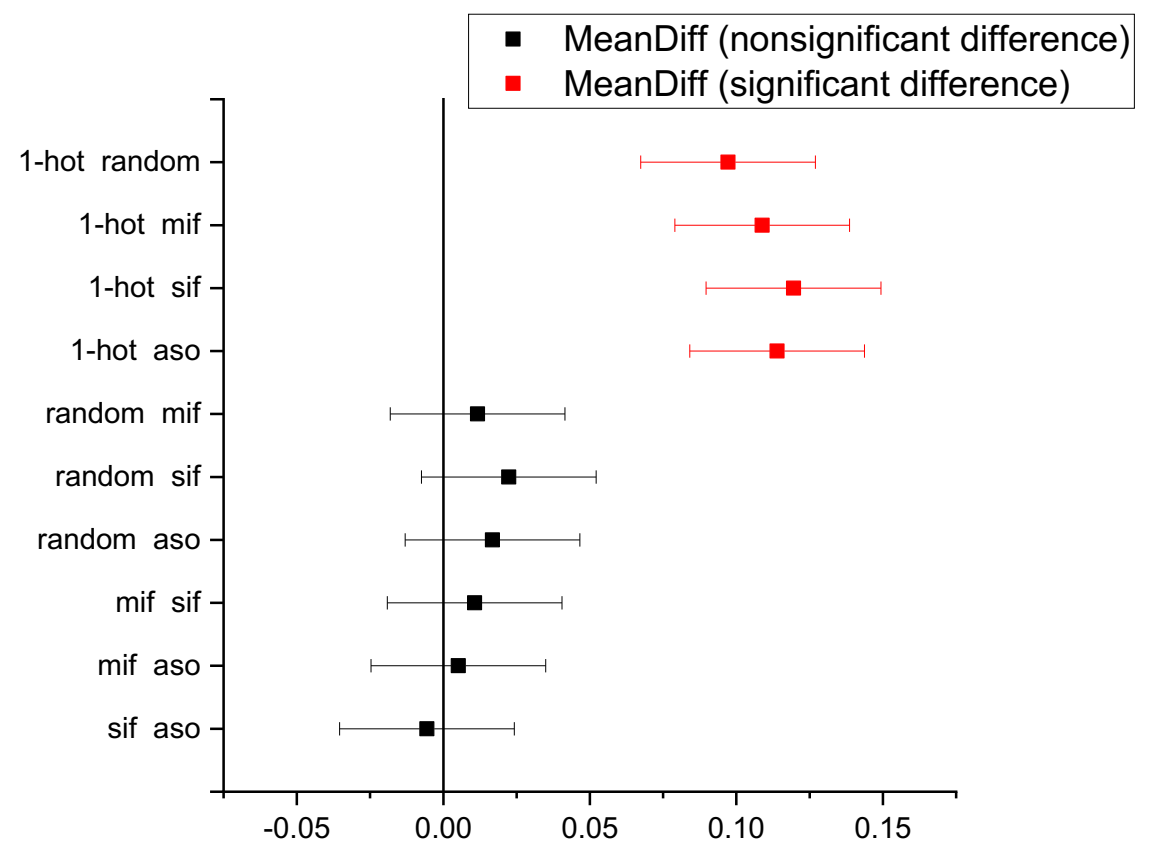

Figure S9. Comparison plot for the randomly selected test sets. 
Analysis for out of sample predictions in the external test set.

Descriptive Statistics
\begin{tabular}{|r|r|r|r|r|r|}
\hline & N Analysis & N Missing & Mean & Standard Deviation & SE of Mean \\
\hline aso & 691 & 0 & 0.25203 & 0.22353 & 0.0085 \\
\hline sif & 691 & 0 & 0.31059 & 0.36484 & 0.01388 \\
\hline mif & 691 & 0 & 0.3396 & 0.3986 & 0.01516 \\
\hline random & 691 & 0 & 0.47189 & 0.43358 & 0.01649 \\
\hline 1-hot & 691 & 0 & 0.38194 & 0.42605 & 0.01621 \\
\hline
\end{tabular}

Figure S10. Descriptive statistics for the MADs of each group for the test set containing out of sample predictions.

\begin{tabular}{|c|c|c|c|c|c|c|c|c|}
\hline \\
\hline \multicolumn{9}{|c|}{ They Test $\rightleftharpoons$} \\
\hline sif aso & 0.05856 & 0.02029 & 4.08096 & 0.03193 & 0.05 & 1 & 0.0032 & 0.11392 \\
\hline mif aso & 0.08757 & 0.02029 & 6.10257 & $1.55189 \mathrm{E}-4$ & 0.05 & 1 & 0.03221 & 0.14293 \\
\hline mif sif & 0.02901 & 0.02029 & 2.02161 & 0.60852 & 0.05 & 0 & -0.02635 & 0.08437 \\
\hline random aso & 0.21985 & 0.02029 & 15.32075 & $2.22045 \mathrm{E}-16$ & 0.05 & 1 & 0.1645 & 0.27521 \\
\hline random sif & 0.16129 & 0.02029 & 11.23979 & 1.93179E-14 & 0.05 & 1 & 0.10593 & 0.21665 \\
\hline random mif & 0.13228 & 0.02029 & 9.21818 & $7.11126 \mathrm{E}-10$ & 0.05 & 1 & 0.07692 & 0.18764 \\
\hline 1-hot aso & 0.12991 & 0.02029 & 9.05291 & 1.53909E-9 & 0.05 & 1 & 0.07455 & 0.18527 \\
\hline 1-hot sif & 0.07135 & 0.02029 & 4.97195 & 0.00401 & 0.05 & 1 & 0.01599 & 0.12671 \\
\hline 1-hot mif & 0.04234 & 0.02029 & 2.95034 & 0.22596 & 0.05 & 0 & -0.01302 & 0.0977 \\
\hline 1-hot random & -0.08994 & 0.02029 & 6.26784 & $9.12664 \mathrm{E}-5$ & 0.05 & 1 & -0.1453 & -0.03459 \\
\hline
\end{tabular}

Sig equals 1 indicates that the difference of the means is significant at the 0.05 level.

Sig equals 0 indicates that the difference of the means is not significant at the 0.05 level.

Figure S11. Tukey post hoc test with pairwise comparisons between groups for the test set containing out of sample predictions. 


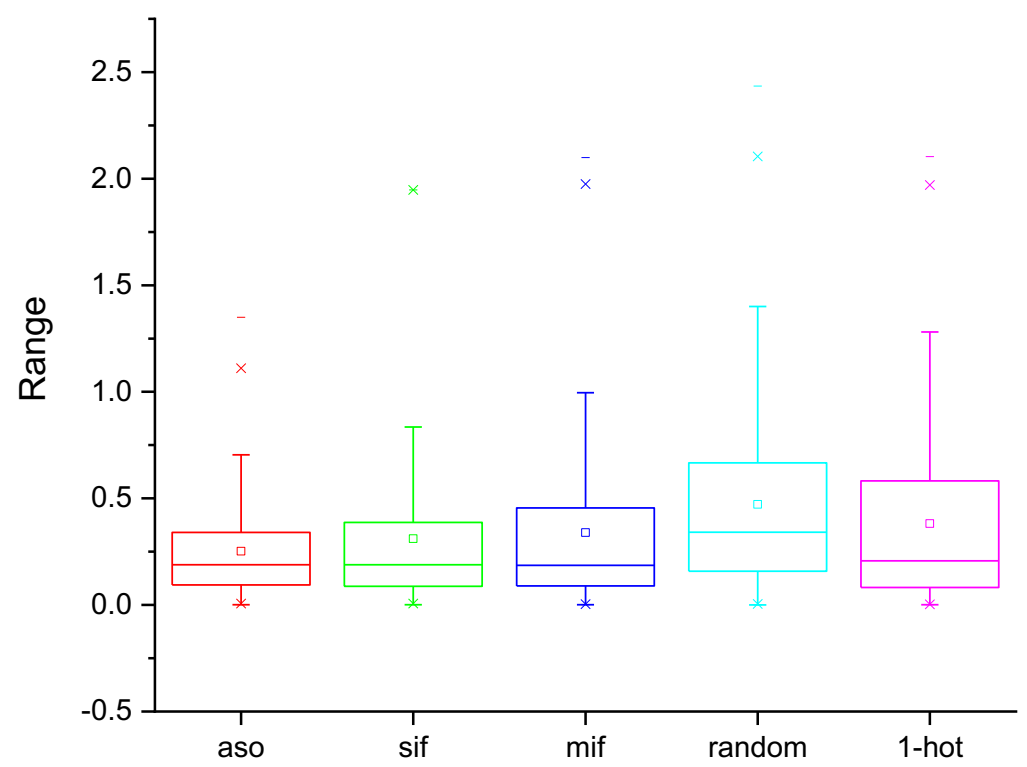

Figure S12. Box plots of MADs for each group for the test set containing out of sample predictions.

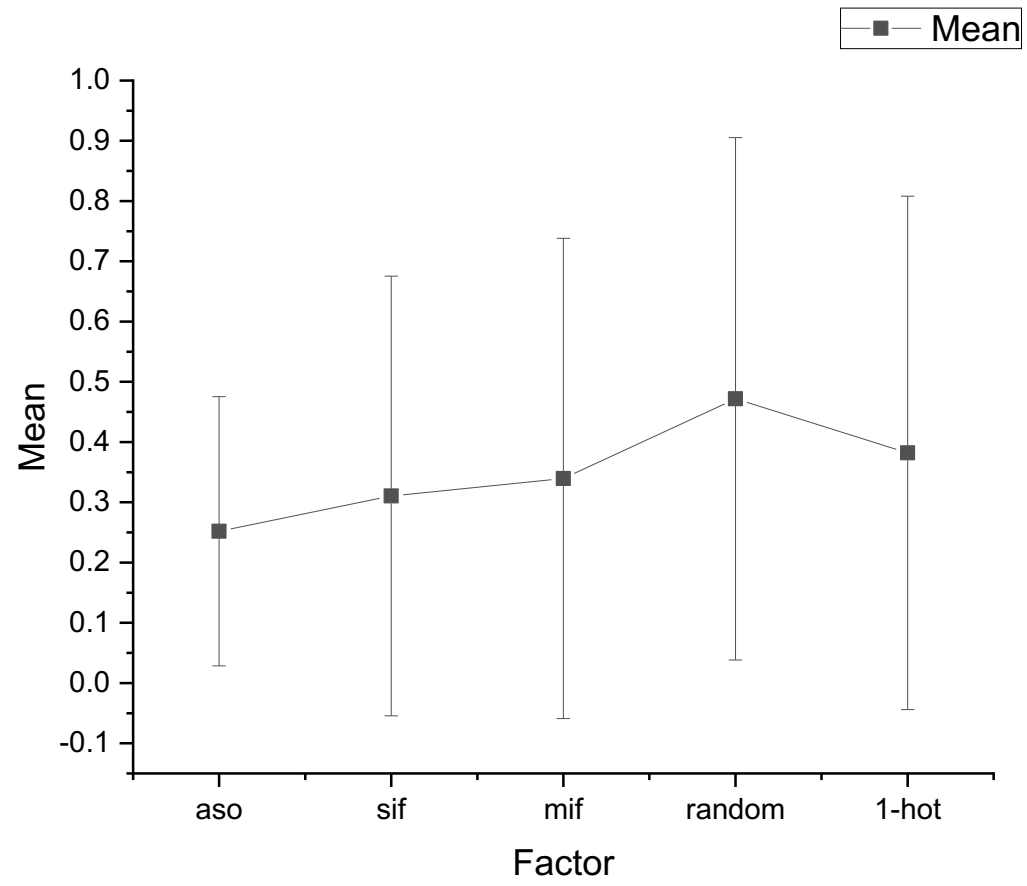

Figure S13. Means plot for the 5 levels used in the ANOVA for the test set containing out of sample predictions. 


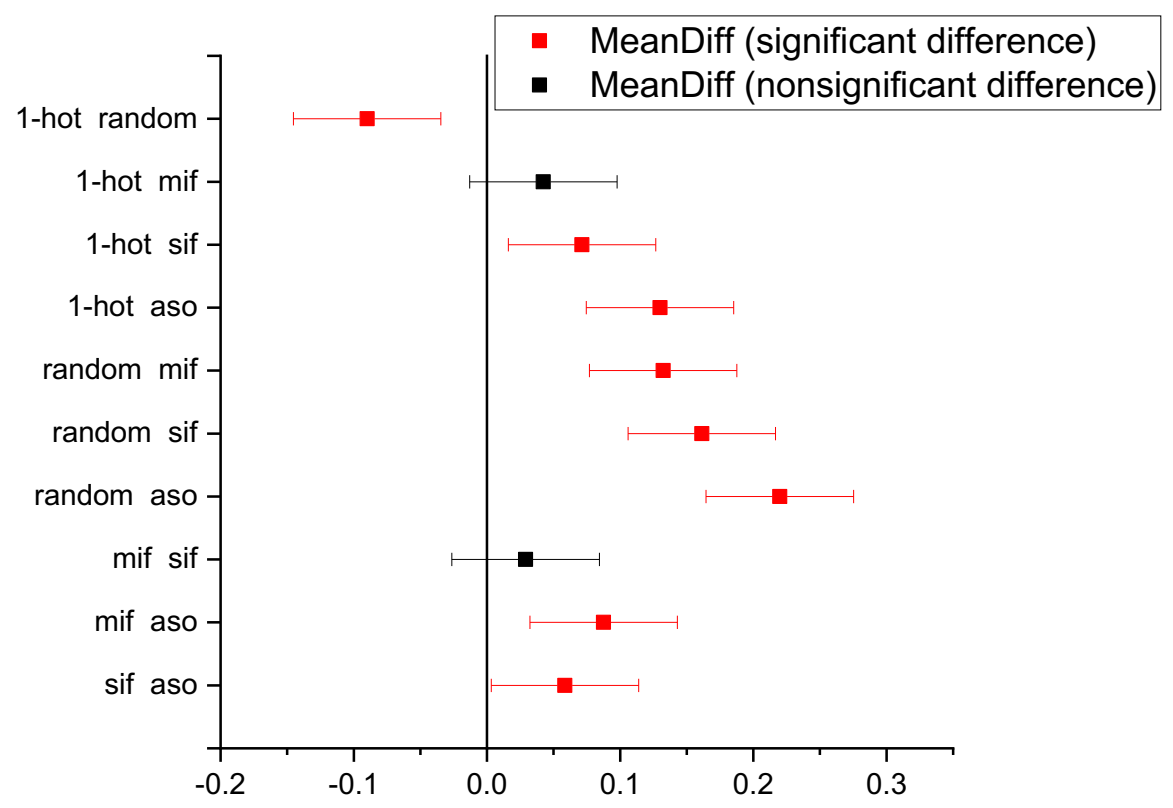

Figure S14. Comparison plot for the test set containing out of sample predictions.

\section{References}

(1) Zahrt, A. F.; Henle, J. J.; Rose, B. T.; Wang, Y.; Darrow, W. T.; Denmark, S. E. Prediction of Higher-Selectivity Catalysts by Computer-Driven Workflow and Machine Learning. Science. 2019, 363, eaau5631.

(2) Zahrt, A. F.; Henle, J. J.; Rose, B. T.; Wang, Y.; Darrow, W. T.; Denmark, S. E. Development of a Computer-Guided Workflow for Catalyst Optimization. Descriptor Validation, Subset Selection, and Training Set Analysis, J. Am. Chem. Soc. 2020, 142, $11578-11592$.

(3) Kubinyi, H. (2008). Comparative Molecular Field Analysis (CoMFA). In Handbook of Chemoinformatics, J. Gasteiger (Ed.)

(4) Pedregosa et al. Scikit-learn: Machine Learning in Python. JMLR. 2011, 12, 28225-2830.

(5) The 109-dimensional vector for randomly generated features was designed to match the dimensionality of the data from the molecular field data after preprocessing. Further, the imine and thiol were set to have the same number of features, and the remainder of the desired number of features was assigned to the catalysts. 